\title{
1 Characterizing the growing microorganisms at species level in 246 anaerobic digesters at Danish wastewater treatment plants: 3 A six-year survey on microbiome structure and key drivers
}

4 Chenjing Jiang ${ }^{1,2}$, Miriam Peces ${ }^{1}$, Martin H. Andersen ${ }^{1}$, Sergey Kucheryavskiy ${ }^{3}$, Marta 5 Nierychlo $^{1}$, Erika Yashiro ${ }^{1}$, Kasper S. Andersen ${ }^{1}$, Rasmus H. Kirkegaard ${ }^{1}$, Liping Hao ${ }^{1}$, 6 Jan Høgh $^{4}$, Aviaja A. Hansen ${ }^{4}$, Morten S. Dueholm ${ }^{1}$, Per H. Nielsen ${ }^{*}$

$7 \quad{ }^{1}$ Center for Microbial Communities, Department of Chemistry and Bioscience, Aalborg 8 University, Aalborg, Denmark.

$9{ }^{2}$ Key Laboratory of Engineering Oceanography, Second Institute of Oceanography, SOA, 10 Hangzhou, China.

$11{ }^{3}$ Department of Chemistry and Bioscience, Aalborg University, Esbjerg, Denmark.

$12{ }^{4}$ Krüger Veolia, Aarhus, Denmark.

$13{ }^{*}$ Corresponding author: Prof. Per Halkjær Nielsen, Center for Microbial 14 Communities, Department of Chemistry and Bioscience, Aalborg University, Fredrik 15 Bajers Vej 7H, 9220 Aalborg, Denmark; Phone+45 9940 8503; E-mail: phn@bio.aau.dk.

\section{Abstract}

Anaerobic digestion (AD) is a key technology at many wastewater treatment plants (WWTPs) for converting surplus activated sludge to methane-rich biogas. However, the limited number of surveys and the lack of comprehensive data sets have hindered a deeper understanding of the characteristics and associations between key variables and the microbiome composition. Here, we present a six-year survey of 46 anaerobic digesters, located at 22 WWTPs in Denmark, which is the largest known study of the microbial ecology of AD at WWTPs at a regional scale. For three types of AD (mesophilic, mesophilic with thermal hydrolysis pretreatment, and thermophilic), we present the typical value range of 12 key parameters including operational variables and performance parameters. The bacterial and archaeal microbiomes were analyzed at species-level resolution using amplicon sequencing in $>1,000$ samples and the new ecosystem-specific MiDAS 3 reference database. We detected 42 phyla, 1,600 genera and 3,584 species in the bacterial microbiome, where $70 \%$ of the genera and $93 \%$ of the species represented uncultivated taxa that were only classified based on MiDAS 3 denovo placeholder taxonomy. More than $40 \%$ of the 100 most abundant bacterial species did not grow in the digesters and were only present due to immigration with the feed sludge. Temperature, ammonium concentration, and $\mathrm{pH}$ were the main drivers shaping the microbiome clusters of the three types of ADs for both bacteria and for archaea. Within mesophilic digesters, feed sludge composition and other key parameters (organic loading rate, biogas yield, and ammonium concentration) correlated with the growing bacterial microbiome. Furthermore, correlation analysis revealed the main drivers for specific species among growing bacteria and archaea, and revealed the potential ecological function of many novel taxa. Our study highlights the influence of immigration on bacterial AD microbiome. Subsetting the growing microbes improves the understanding of the 
diversity and main drivers of microbiome assembly, and elucidates functionality of specific species-level microorganisms. This six-year survey provides a comprehensive insight into microbiome structure at species level, engineering and ecological performance, and a foundation for future studies of the ecological significance/characteristics and function of the novel taxa.

\section{Introduction}

Anaerobic digestion (AD) is successfully employed worldwide to convert organic feedstock into biogas by anaerobic mixed microbial communities. As a key technology at wastewater treatment plants (WWTP), AD is used to reduce and stabilize the primary and waste-activated sludge by generating methane for bioenergy production. Moreover, $\mathrm{AD}$ can be used as a platform for the recovery of value-added compounds (e.g., phosphorus, nitrogen, volatile fatty acids) [1,2]. Thus, it is an important step in the development of circular economy at the WWTPs. The conversion of organic feedstock is carried out by the $\mathrm{AD}$ microbiome, a complex network of hydrolyzing and fermenting bacteria, specialized acidogenic and acetogenic syntrophs, and methanogenic archaea [3], which is shaped by stochastic (birth-death immigration) and deterministic (microbial competition, operation and environment) factors $[4,5]$. Hence, a good understanding of the microbial ecology in digesters is essential for informed control and manipulation of the process for optimal performance.

$\mathrm{AD}$ harbours a complex microbial network which is ideal for identifying diversity trends in constrained microbial community structures. Research has shown that the operational parameters, including temperature, substrate type, organic loading rate (OLR), and sludge retention time (SRT) are vital factors for determining the microbiome structure [6-12]. Other parameters, such as ammonia concentration and salinity, are also thought to be significant drivers shaping the microbiome [7,13-15]. Additionally, the microorganisms immigrating with the feed sludge should not be overlooked. Most of them do not grow or contribute to the ecological functions in the system, but they still account for a significant fraction of sequencing reads identified by $16 \mathrm{~S}$ rRNA gene amplicon sequencing $[7,9,13]$.

However, most of these findings are based on investigations across various AD substrate types, such as manure, food waste, and wastewater sludge, where large differences in growth conditions are observed. Whether the same drivers are also important among digesters at WWTPs is unclear. The AD performance can be highly variable between different WWTPs, but how this links to different microbiomes and growth conditions is poorly described for full-scale systems.

The quantitative relationships between specific microorganisms and key parameters in $\mathrm{AD}$ can be evaluated by multiple linear regression (MLR). Most studies have focused on linear associations between methanogenic populations (i.e., characterized by the $\mathrm{mcr} A$ gene) and specific methanogenic activities [17-20]. However, the traditional MLR fails when the number of predictors is comparable to, or larger than, the number of observations, and when there is high collinearity in predictors. Projection-based methods for analysis of multivariate data, such as Partial Least Squares (PLS) regression, stand as 
promising techniques to evaluate the links between specific microorganisms and key parameters, lessening the shortcomings of traditional methods [21].

To provide insightful links between the AD microbiome and its performance, it is crucial to obtain a high phylogenetic resolution and good taxonomic classification at all ranks. A high phylogenetic resolution can be obtained by using amplicon sequence variants (ASVs) $[22,23]$ instead of operational taxonomic units (OTUs) typically clustered at $97 \%$ similarity thresholds, and by using an ecosystem-specific, high-quality 16S rRNA gene reference database for taxonomic classification. We have developed MiDAS 3, a comprehensive ecosystem-specific reference database for activated sludge and anaerobic digesters which provides a taxonomic classification at all ranks for all sequences based on an improved and automated classification system (AutoTax) [24,25]. The MiDAS 3 reference database is based on full-length 16S rRNA gene ASVs (FL-ASVs) obtained from Danish WWTPs and digesters, but can be applied to similar systems worldwide [24]. MiDAS 3 improves the classification of prokaryotic microorganisms found in AD compared to other public reference databases (SILVA [26], Greengenes [27], and RDP [28]), which lack reference sequences for many taxa and high taxonomic resolution, often resulting in poor classification (Figure S1). Application of MiDAS 3 for the study of AD communities offers a possibility of finding the link between identity and function of species-level taxa. Species names provide stable taxa identifiers independent of the data set, thus, allowing cross-study comparisons.

The aims of our study are threefold. Firstly, we describe the typical operational parameters and performance values of three different types of $\mathrm{AD}$ at WWTPs (i.e., mesophilic $\mathrm{AD}$, mesophilic $\mathrm{AD}$ with pre-treatment (thermal hydrolysis) of waste activated sludge, and thermophilic AD). Secondly, we present the microbial communities in the $\mathrm{AD}$ systems (with focus on the growing microbes), for the first time at species level, and make this publicly available on the MiDAS website (https://www.midasfieldguide.org/guide). And thirdly, by focusing on species-level microbiome, we analyse the correlations between key AD parameters and microbiome structure in mesophilic digesters, which are the most common digesters in Denmark at WWTPs.

\section{Methods}

\section{Anaerobic digesters and sample collection}

The survey was conducted during the period $2011-2016$ in 46 anaerobic digesters at 22 WWTPs across Denmark, which were operated under mesophilic (MAD), mesophilic with thermal hydrolysis pretreatment of feedstock (THP-MAD), or thermophilic (TAD) conditions (see Table S1 for information of digesters). During the six years of survey, all plants reported minor fluctuations in substrate amounts and composition, but no major changes of operating conditions were introduced, except for Aaby and Aalborg East, which switched from mesophilic to thermophilic operation (Table S1). A total of more than 50,000 observations, including operational, physicochemical, and performance parameters, except volatile fatty acids (VFAs), were obtained from the records of 
individual plants. Each key variable had at least 1,087 observations, except VFAs (Table 1).

The digester sludge samples were obtained 2-4 times a year during the investigation period, and the VFA samples were collected 2-5 times from each studied digester during 2016. All samples were transported to the laboratory within $24 \mathrm{~h}$ and processed immediately upon arrival. After homogenization, the biomass samples were stored as 2 $\mathrm{mL}$ aliquots at $-80^{\circ} \mathrm{C}$ before DNA extraction. Samples for VFA analysis were filtered with $0.22 \mu \mathrm{m}$ filters (Frisenette, Knebel, Denmark) and stored at $-20^{\circ} \mathrm{C}$ until analysis, which is described elsewhere [29].

\section{DNA extraction, 16S rRNA gene amplicon sequencing, and bioinformatics processing}

The microbial communities of a total of 1,010 AD sludge samples (418 for archaea and 592 for bacteria) were analyzed using $16 \mathrm{~S}$ rRNA gene amplicon sequencing. $50 \mu \mathrm{AD}$ sample were used for DNA extraction with the FastDNA ${ }^{\circledR}$ Spin Kit for soil (MP Biomedicals, Solon, OH, USA), following the optimized protocol for anaerobic digesters by Kirkegaard et al. [30]. The library preparation for $16 \mathrm{~S}$ rRNA amplicon sequencing was performed as described in Kirkegaard et al. [10], targeting the V1-3 variable regions for bacteria and V3-5 variable regions for archaea. The bacterial primers used were $27 \mathrm{~F}$ (AGAGTTTGATCCTGGCTCAG) [31] and 534R (ATTACCGCGGCTGCTGG)[32], which amplify a DNA fragment of $\sim 500 \mathrm{bp}$ of the $16 \mathrm{~S}$ rRNA gene (V1-3). The archaeal primers used were 340F (CCCTAHGGGGYGCASCA) [33] and 915R (GWGCYCCCCCGYCAATTC) [33], which amplify a DNA fragment of $\sim 560 \mathrm{bp}$ of the $16 \mathrm{~S}$ rRNA gene (V3-5). The amplicon libraries were paired-end sequenced $(2 \times 300 \mathrm{bp})$ on the Illumina MiSeq as described by Albertsen et al. [34].

The archaeal and bacterial read data were analyzed separately using USEARCH (v.11.0.667) [35]. For the V1-3 amplicons raw fastq files were filtered for phiX sequences using -filter_phix, trimmed to $250 \mathrm{bp}$ using -fastx_truncate -trunclen 250 , and quality filtered using -fastq_filter with -fastq_maxee 1.0. The sequences were dereplicated using -fastx_uniques with -sizeout -relabel Uniq. ASVs were generated using UNOISE3 [36], and ASV-tables were created by mapping the raw reads to the ASVs using -otutab with the -zotus and -strand both options. Taxonomy was assigned using the MiDAS 3 reference database $[24,25]$ using sintax with the -strand both and -sintax_cutoff 0.8 [37]. The V3-5 amplicon data were analyzed in the same way except that only the reverse read was used and the primer binding site was removed during the trimming using -fastx_truncate stripleft 18 -trunclen 250.

\section{Data processing and statistical analysis}

Downstream statistical analyses and visualization were mostly performed in the $\mathrm{R}$ environment (v3.6.2) [38] using ampvis2 (v2.5.8) [34] and ggplot2 (v3.2.1) [39], unless indicated otherwise. Non-parametric dunn.test was used to identify significant differences between $\mathrm{AD}$ types. The correlations between all the variables were explored by Spearman correlation, where correlations greater than \pm 0.5 and false discovery rate (FDR) corrected $P>0.05$ were visualized in Gephi (v0.9.2) [40], using Force Altas2 and manual tweaking 
to generate the network. For sequence data, samples were randomly subsampled to 10,000 sequences per sample, yielding a final dataset of 402 archaeal and 564 bacterial samples. For the growing bacteria datasets, after removing the non-growing ASVs from ASVtable, samples were also randomly subsampled to 10,000 sequences per sample for downstream analysis and comparison. Boxplot and heatmaps were made by the amp_boxplot and amp_heatmap function in ampvis2. Alpha diversity was calculated by amp_alphadiv function in ampvis2. The linear regression between alpha diversity (using Shannon's index) and each operational and performance variable was used to pick the key variables most correlated. Weighted uniFrac distance, calculated by beta_diversity.py script in QIIME (v1.9.0) [41], was applied for all beta diversity comparisons. For ordination visualizations, the non-metric multidimensional scaling (NMDS) was performed by amp_ordinate in ampvis 2 to show the dissimilarities of microbial profiles. Based on weighted uniFrac distance matrix, ANOSIM was applied to assess similarities for categorical variables using compare_categories.py in QIIME with 999 permutations. A PERMANOVA analysis using adonis in QIIME was used to describe the strength and significance for continuous variables. The significant difference of species between two groups of feed sludge was explored by Wilcoxon rank-sum test.

PLS regression was performed using $\mathrm{R}$ package mdatools v0.10.1 [21] to validate quantitative relationship between operational and performance parameters, and the microbial community, as well as to identify specific microbes which correlate to each variable the most. All bacterial species with median relative abundance $>=0.01 \%$ and archaeal ASVs with median relative abundance $>=0.05 \%$ were used to perform the PLS analysis. The model was trained using all samples and validated by segmented crossvalidation $(\mathrm{CV})$ with systematic splits (venetian blinds). Determination coefficient $\left(\mathrm{R}^{2}\right)$ and root mean square error (RMSE) were used to assess performance of the model. The contribution of individual predictors was evaluated using regression coefficients and corresponding inferential analysis carried out by Jack-Knifing approach [42].

\section{Results and discussion}

\section{Characterization of key parameters of AD}

Key operational and performance parameters of the 46 anaerobic digesters during the sixyear survey are summarized in Table 1. The digesters are classified into three types, based on the operational temperature and pretreatment of the feed sludge. MAD is the most common configuration (78\% of all digesters) followed by TAD (15\%) and THP-MAD (7\%). The most common digester type is single-stage continuously stirred tank reactor (CSTR). The anaerobic digesters surveyed were running stably without major process complications for six years, therefore common ranges of operation and performance conditions are described for each digester type. As presented in Table $\mathbf{1}$ and described below, values of several environmental parameters are very different from other AD systems treating manure, crops, food waste, and industrial waste [7,11,15,43-47], with generally lower or much lower values of total ammonia nitrogen (TAN) and level of VFAs. 
206 The median temperature values of the three types of digesters were $38.0^{\circ} \mathrm{C}, 38.6^{\circ} \mathrm{C}$, and

$20753.6^{\circ} \mathrm{C}$, for MAD, THP-MAD, and TAD, respectively. Other operational variables (OLR

208 and SRT) and the performance parameters ( $\mathrm{pH}$, TAN, alkalinity, TS, VS, biogas yield,

209 and methane content) were found to be significantly different across all three types of AD

210 (Figure S2). For more details on the description of each parameter, please see Additional

211 file 2. In general, the same overall correlations between operational and performance

212 parameters across digesters treating different types of substrates could also be observed

213 specifically for digesters among WWTPs. Strong positive correlations (Spearman, $r>0.7$,

214 false discovery rate (FDR) $P<0.05)$ were observed between TAN and TS or VS, OLR

215 and alkalinity, and methane production and SRT (Figure S3). Strong negative

216 correlations between the OLR and methane production and biogas yield were also

217 revealed (Spearman, $r>0.65$, FDR $P<0.05$ ), and between methane production and TAN

218 (Figure S3), indicating that these operational variables are linked to the performance of

219 the digesters. It is interesting that VFAs only related weakly to SRT and the ratio of VS

220 to TS, which have previously been considered as important variables [48]. This may be

221 due to a general low concentration range in the digesters and low loading.

222 Table 1. Operational variables and performance parameters: Intervals and median 223 values for ADs at WWTPs in Denmark.

\begin{tabular}{|c|c|c|c|c|c|c|c|c|}
\hline \multirow[t]{2}{*}{ Category } & \multirow[t]{2}{*}{ Variable } & \multirow[t]{2}{*}{ Unit } & \multicolumn{2}{|c|}{ MAD* } & \multicolumn{2}{|c|}{ THP-MAD* } & \multicolumn{2}{|c|}{ TAD* } \\
\hline & & & Interval $^{1}$ & Median & Interval & Median & Interval & Median \\
\hline \multirow{3}{*}{$\begin{array}{c}\text { Operational } \\
\text { variables }\end{array}$} & Temperature & ${ }^{\circ} \mathrm{C}$ & $35.6 \sim 39.9$ & 38.0 & $38.2 \sim 39.0$ & 38.6 & $51.1 \sim 55.40$ & 53.6 \\
\hline & $\mathrm{OLR}^{2}$ & $\mathrm{~kg} \mathrm{VS} / \mathrm{m}^{3} \cdot \mathrm{d}$ & $0.84 \sim 1.13$ & 0.96 & $1.66 \sim 2.30$ & 2.04 & $1.63 \sim 2.49$ & 2.15 \\
\hline & SRT $^{*}$ & Day & $24.8 \sim 35.6$ & 29.4 & $27.3 \sim 34.9$ & 30.1 & $15.8 \sim 20.7$ & 17.3 \\
\hline \multirow{11}{*}{$\begin{array}{c}\text { Performance } \\
\text { parameters }\end{array}$} & TS* & $\mathrm{g} / \mathrm{L}$ & $21.1 \sim 38.4$ & 31.0 & $41.1 \sim 65.9$ & 44.8 & $31.2 \sim 39.0$ & 35.8 \\
\hline & VS* & $\mathrm{g} / \mathrm{L}$ & $12.1 \sim 19.9$ & 16.2 & $25.9 \sim 34.0$ & 27.7 & $21.0 \sim 25.5$ & 23.6 \\
\hline & VS & $\mathrm{TS} \%$ & $56.0 \sim 61.6$ & 58.6 & $54.0 \sim 63.5$ & 60.2 & $57.0 \sim 60.9$ & 58.0 \\
\hline & $\mathrm{pH}$ & - & $7.06 \sim 7.38$ & 7.19 & $7.64 \sim 7.86$ & 7.75 & $7.50 \sim 7.80$ & 7.70 \\
\hline & TAN & $\mathrm{mg} \mathrm{N} / \mathrm{L}$ & $603 \sim 972$ & 745 & $2691 \sim 3100$ & 2888 & $1070 \sim 1430$ & 1215 \\
\hline & Alkalinity & $\mathrm{mM}$ & $50.0 \sim 73.0$ & 60.8 & $148 \sim 186$ & 168.6 & $67.9 \sim 87.7$ & 78.4 \\
\hline & Total VFA* & $\mathrm{mM}$ & $0.28 \sim 1.11$ & 0.50 & $0.45 \sim 2.34$ & 0.73 & $0.92 \sim 2.18$ & 1.30 \\
\hline & Acetate & $\mathrm{mM}$ & $0.10 \sim 0.40$ & 0.21 & $0.25 \sim 0.44$ & 0.30 & $0.53 \sim 1.18$ & 0.79 \\
\hline & Biogas yield $^{2}$ & $\mathrm{Nm}^{3} / \mathrm{kg} \mathrm{VS}$ & $0.39 \sim 0.53$ & 0.46 & $0.38 \sim 0.56$ & 0.49 & $0.25 \sim 0.36$ & 0.29 \\
\hline & Methane content & $\%$ & $61.0 \sim 63.5$ & 61.7 & $63.0 \sim 65.0$ & 65.0 & $53.7 \sim 64.3$ & 57.6 \\
\hline & Methane production ${ }^{3}$ & $\mathrm{Nm}^{3} / \mathrm{m}^{3} \cdot \mathrm{d}$ & - & 0.27 & - & 0.65 & - & 0.36 \\
\hline
\end{tabular}

$224{ }^{1}$ Interval shows the range of first quantile and third quantile of each variable.

$225{ }^{2}$ OLR and biogas yield are normalized using an average volatile solids value of influent feed (74.5\%).

$226{ }^{3}$ Methane production is calculated on median value of biogas yield, OLR, and methane content.

$227 * \mathrm{MAD}=$ mesophilic, THP-MAD $=$ mesophilic with thermal hydrolysis pretreatment, TAD $=$ thermophilic, OLR $=$ organic loading rate, $\mathrm{SRT}=$ solids retention time, $\mathrm{TS}=$ total solids, $\mathrm{VS}=$ volatile solids, $\mathrm{TAN}=$ total ammonia nitrogen, Total VFA $=$ total volatile fatty acid.

231 Like many other full-scale plants, running at low OLR and long SRT of the digesters 232 surveyed, which is referred as "suboptimal" operational conditions, may lead up to a $30 \%$ 233 profitability loss [46,49]. Increasing the OLR seems promising, but there may be a 234 number of operational problems which need to be considered, such as foaming and 235 acidosis, due to the imbalance between operational and microbial processes. Thus, a better 236 understanding of microbial communities and their function may help to control or 237 manipulate the processes that decrease the potential risks of operational failures. 


\section{Bacterial and archaeal microbiomes}

We obtained 33,047 bacterial and 878 archaeal unique ASVs, which were classified and assigned using sintax and the MiDAS 3 database. Thus, a total of 42 phyla, 1,600 genera, and 3,584 species were detected in the bacterial microbiome, where 1,117 (70\%) genera and 3,336 (93\%) species were novel and could only be assigned genus and species name based on the MiDAS 3 denovo placeholder taxonomy. For archaea it was not possible to analyze the methanogenic archaea at the species level because the phylogeny of most of these species cannot be resolved using the 16S rRNA gene, even with full-length sequences [24]. As a result, only 26 species were classified and most of the archaeal population are shown at ASV level.

$\mathrm{AD}$ at WWTPs are complex systems, as they receive a substantial amount of microorganisms via feed streams (primary sludge, PS, or surplus activated sludge, AS). Many of these microorganisms are not growing in the digester, presumably inactive or dying off $[10,13,50]$. The growing and non-growing microorganisms were identified according to the ratio of read abundances in digester and feed as described by Kirkegaard et al. [10]. The bimodal distribution of ratios was split at a ratio of around 10 (Figure S4), showing two clearly separated groups of ASVs. The group with a ratio $>10$ represents ASVs enriched in digesters compared to the feed sludge, here designated as "growing microorganisms". The group with a ratio $<10$ represents ASVs with unchanged or lower relative read abundance in digesters, compared to the feed sludge, here designated as "non-growing microorganisms". Thus, combined with median relative abundance across samples in each type of $\mathrm{AD}$, the total ASVs $(>0.01 \%$ median relative abundance) were divided into four groups, growing/non-growing ASVs with high abundance $(>0.1 \%)$ and growing/non-growing ASVs with low abundance $(<0.1 \%)$. It was observed that the growing highly abundant ASVs only accounted for $7.6 \%, 23.2 \%$, and $9.4 \%$ of the total ASV counts in MAD, THP-MAD, and TAD, respectively (Fig. 1). However, the proportion of relative abundances of these growing highly abundant ASVs were large, at $38.8 \%, 85.3 \%$, and $50.9 \%$ in MAD, THP-MAD, and TAD, respectively. This suggests that the performance and functionality of AD might be driven by only a small number of the microbial phylotypes detected by amplicon sequencing.

The five most abundant bacterial phyla were Firmicutes, Proteobacteria, Chloroflexi, Actinobacteria, and Bacteroidetes, accounting for $75.7 \%$ (median value) of all amplicon sequences across all samples, and these phyla are typical for digesters at WWTPs $[7,15,43,51-54]$. However, the three types of $\mathrm{AD}$ showed variations in the dominant bacterial taxa, especially at genus level (Figure S5). Among the 25 most abundant species, 11 species in MAD and 9 species in TAD belonged to the group of non-growing microorganisms (Fig. 2A and 2B). These included species in genera belonging to the polyphosphate-accumulating organisms (PAO) Tetrasphaera, the putative PAO Dechloromonas [55], the filamentous genus $\mathrm{Ca}$. Microthrix [56], and the genera Romboutsia, and Trichococcus. These all belong to the top-most abundant reported genera in activated sludge in Danish WWTPs [25], thereby indicating carry-over to the digesters with the feed sludge. Since the top100 species in MAD and TAD, respectively, are very similar across the digesters, these lists can be used as a representative reference 
of abundant growing and non-growing organisms in digesters at WWTPs across the world (Figure S6 and S7). These results demonstrate that surprisingly many, 44\% and 54\% of the species were non-growing in MAD and TAD, respectively. The top 25 species in THP-MAD all belonged to growing microorganisms in good agreement with the presence of THP pretreatment, which causes a decay of essentially all organisms coming with the feed sludge (Figure S8A).

The growing microorganisms are considered to be responsible for the most important ecological functions within AD. Among the dominant growing bacterial species there were many known fermenters, such as species belonging to the genera Thermovirga, Ca. Fermentibacter, and Leptolinea in MAD, and Coprothermobacter and Acetomicrobium in TAD. There were also syntrophic bacteria, such as members of $C a$. Cloacimonas [57] and Syntrophorhabdus in MAD. However, a large fraction of the most abundant growing species were novel taxa without any known function. They were identified by MiDAS 3 species-level taxonomy and given robust placeholder names until characterized in more detail, enabling across-study comparisons of $\mathrm{AD}$ microbiome at high taxonomic resolution [24]. Due to the high relative abundance, some genera are of special interest: midas_g_12 (family Prolixibacteraceae), midas_g_19 (family Bacteroidetes vadinHA17), midas_g_156 (family Anaerolineaceae), and midas_g_789 (family Anaerolineaceae) in MAD; midas_g_88 (family Syntrophomonadaceae), midas_g_112 (order MBA03), and midas_g_16 (family Lentimicrobiaceae) in TAD, and midas_g_13 (order D8A-2) in THPMAD. Some of these genera encompass very abundant species, especially in the family Anaerolineaceae (up to $8 \%$ median abundance): midas_s_156, midas_s_876, midas_s_956, midas_s_1462, midas_s_467, midas_s_1625. These abundant and novel taxa should be investigated in future studies, as their physiology and ecological role in $\mathrm{AD}$ are completely unknown while likely important.

Moreover, compared with MiDAS 2 taxonomy (which was the curated version of Silva taxonomy) [58], MiDAS 3 provides a much higher resolution to classify sequences and introduces species-level names for the first time for the abundant microorganisms in AD ecosystem. For example, genus T78 (family Anaerolineaceae) in MiDAS 2 encompassed sequences that are split into midas_g_156 and midas_g_467 in MiDAS 3, both being the abundant genera mentioned above. These genera are both diverse, each having three abundant species present in MAD (Figure S9). Also, $\mathrm{Ca}$. Cloacimonas and Pelotomaculum and the newly discovered syntrophic genus midas_g_995 [29] had high species diversity as well, with several abundant species with random distribution (Figure S9).

Euryarchaeota was the dominant archaea in the digesters (99.9\%, median value). The acetoclastic genus Methanothrix (previously named Methanosaeta) dominated in MAD (71.8\%) and THP-MAD (93.8\%), whereas the genera Methanothermobacter (70.7\%) and Methanosarcina (24.8\%) dominated in TAD (Figure S10B). Methanosarcina was in very low abundance in MAD (0.1\%) and THP-MAD (0.01\%), in contrast to other mesophilic full-scale studies of manure-based $\mathrm{AD}$ where it was dominant [7,53,59]. However, the low concentrations of VFAs $(<1 \mathrm{mM})$ in the mesophilic AD may explain why Methanothrix dominated [60]. The hydrogenotrophic methanogenic Methanoculleus was 
the predominant genus (4.2\%) in THP-MAD, which is in line with other lab-scale and pilot-scale THP digesters studies [61,62].

Acetoclastic and hydrogenotrophic methanogenic species/ASVs were quite abundant in all the types of AD (Fig. 2C, 2D, and Figure S8B). g_Methanothrix_ASV1 was dominant in both MAD and THP-MAD, followed by midas_s_1190 (genus Methanolinea) and g_Methanolinea_ASV6 in MAD and midas_s_880 (genus Methanoculleus) in THPMAD. Methanothermobacter tenebrarum and Methanosarcina thermophila species were the second most abundant species in TAD (Fig. 2).

\section{Microbial diversity in different AD types}

The use of common measures for richness and diversity in the digesters surveyed has only limited value, as abundant immigrating bacteria, likely inactive or dying off without any functional role in the systems, will influence the diversity measures and produce misleading results. This is illustrated by comparing the diversity measures calculated for all bacteria and for the growing bacteria only (Fig. 3A). When the non-growing fraction was removed, the median values of observed ASVs decreased from 1935 to 928, 1486 to 534, and the median values of Shannon index from 6.22 to 5.11 and 5.59 to 4.33 in MAD and TAD, respectively (Fig. 3A). THP-MAD only had a minor change in observed ASVs (from 832 to 741 ) and the median Shannon index (4.47 and 4.63, respectively), reflecting, as expected, that these communities were not strongly influenced by immigration. The adjusted diversity measures showed the same order of magnitude decrease for archaea (Figure S11) with THP-MAD between MAD and TAD. The measures also showed that higher temperature harbored fewer number of microbes in accordance to other full-scale surveys [15,51], but that the exact values are strongly dependent on the inclusion of the immigrating microbes. Higher alpha diversity measures for bacterial communities compared with archaea is in agreement with other full-scale WWTPs studies [15,43,51]. The diversity in thermophilic AD has been shown to be lower than in mesophilic digesters [63-65], which is also supported by our data.

The total bacterial (including growing and non-growing fraction) and archaeal microbiome seemed relatively stable in each digester across all 22 WWTPs during the six-year survey as indicated by tight clustering as visualized by non-metric multidimensional scaling (NMDS) (Figure S12). This is also reported from other timeseries studies of full-scale digesters mainly treating manure, agricultural waste, and municipal solid waste $[7,43]$, suggesting that overall stable microbiomes are common in full-scale digesters during steady-state operation. However, as a major part of the microorganisms are immigrants, they may strongly affect the betadiversity measures. Therefore, it is important to compare the diversity of both the total and the growing fraction of the population. The dissimilarity among plants seemed the same considering the community structure of the total and growing bacteria in MAD (ANOSIM; Total bacteria: $\mathrm{R}=0.65, P=0.001$; Growing bacteria: $\mathrm{R}=0.63, P=0.001)$ and THP-MAD (ANOSIM; Total bacteria: $\mathrm{R}=0.45, P=0.001$; Growing bacteria: $\mathrm{R}=0.49, P=0.001$ ). However, the growing bacterial community in TAD became more similar across plants compared to the total bacterial population (ANOSIM; Total bacteria: $\mathrm{R}=0.64, P=0.001$; Growing bacteria: $\mathrm{R}=0.40, P=0.001)$. This shows that the inclusion of non-growing 
bacteria in microbiome analyses of TAD may lead to misleading results and erroneous conclusions.

Analysis of beta diversity of the communities present in different AD types revealed three distinct clusters corresponding to MAD, THP-MAD, and TAD (Fig. 3B and Figure S13A). Clear separation dictated by AD type was evident for all bacteria (ANOSIM: $\mathrm{R}=$ $0.95, P=0.001$ ), the growing bacteria (ANOSIM: $\mathrm{R}=0.97, P=0.001$ ), as well as the archaeal microbiome (ANOSIM: $\mathrm{R}=0.83, P=0.001$ ), reflecting the huge effects of operational conditions on the resulting variation in the microbiomes. Permutational multivariate analyses of variance showed that TAN contributed to shaping the structure of the total bacterial microbiome (adonis: $\mathrm{R}^{2}=32 \%, P=0.001$ ) (Figure S13B), which has also been observed in full-scale digesters treating different kinds of substrates [7]. In contrast to the bacterial microbiome, the overall structure of the archaeal microbiome was separated mainly by temperature (adonis: $\mathrm{R}^{2}=66 \%, P=0.002$ ), with a separate cluster for THP-MAD alongside MAD (Figure S13C). $\mathrm{pH}$ was the second factor influencing the archaeal microbiome (adonis: $\mathrm{R}^{2}=27 \%, P=0.002$ ), which may explain the separated cluster of THP-MAD from MAD (Figure S13D).

\section{Main drivers of MAD microbiome}

Since most digesters surveyed were MAD, we further applied the correlation analysis between key parameters and microbial diversity and structure to determine the main drivers, with special focus on the growing bacterial microbiome, as non-growing microorganisms may mask the influence of key drivers on the active microbiome in correlation analyses. In general, bigger difference was observed on linear regression of key parameters against alpha diversity between the total and growing bacterial microbiomes, compared with permutational multivariate analysis of betadiversity (Table S2 and Table S3).

It is well-known that temperature is a very important factor for shaping the microbial diversity and community structure in full-scale digesters [7,15,43], but it is less clear to what extent it is for mesophilic AD at WWTPs. In our study, the temperature range in MAD was small (35.6-39.9) and was only considered to be most important to the total bacterial alpha diversity in $\operatorname{MAD}(25 \%$, linear regression, FDR $P<0.001$, Table S2), but not the alpha diversity of growing bacteria $(16 \%$, FDR $P<0.001)$. This indicates that temperature may not be the most important factor in MAD. Instead, the correlation coefficient of OLR improved significantly by subsetting the growing bacterial alpha diversity $(31 \%, P<0.001)$ compared to the total bacterial alpha diversity $(9 \%, P>0.05)$. OLR also shaped the microbiome structure (beta diversity) of growing bacteria (adonis: $\mathrm{R}^{2}=21 \%, P=0.001$, Table S3). Although OLR is widely accepted as a deterministic factor for any type of AD microbiome [66-69], our study strengthened it when OLR was only correlated with growing bacteria. Moreover, the biogas yield exhibited strong correlation with the growing bacterial microbiome both on alpha diversity $(46 \%, P<$ 0.001 ) and beta diversity (adonis: $\mathrm{R}^{2}=31 \%, P=0.001$ ), as well as archaeal beta diversity (adonis: $\mathrm{R}^{2}=23 \%, P=0.008$ ), supporting the observation that $\mathrm{AD}$ performance depends on the activity of the microbiome [70]. Similarly, TAN was observed to be more correlated to growing bacterial alpha diversity compared with the total bacterial 
population (Table S2). Regarding the archaeal microbiome, no strong correlation was found between parameters and alpha diversity in MAD. However, apart from biogas yield, acetate concentration (adonis: $\mathrm{R}^{2}=18 \%, P=0.04$ ) was also found to have significant correlation with the archaeal microbiome structure.

Samples from MAD digesters treating only surplus AS and without PS (Fornaes, Mariagerfjord, and Soeholt) formed a small separate cluster, compared to MAD treating a mixture of both types of substrates for all bacteria (ANOSIM: $\mathrm{R}=0.44, P=0.001$, Figure S14A) and for the growing bacteria (ANOSIM: $\mathrm{R}=0.57, P=0.001$, Fig. 3C). The higher dissimilarity of growing bacteria in MAD supports the observation that substrate characteristics (e.g., biodegradability, composition, concentration) from PS shape the growing bacterial community structure [7,15]. This is different from the growing bacteria in the three types of $\mathrm{AD}$, which is mainly driven by operational parameters (Fig. 3B). While for all bacterial communities, the relative abundance of 18 of the 25 most abundant species showed a significant difference between the two clusters depending on the feed sludge (Wilcoxon rank-sum test, $P<0.05$, Figure S14B). Nongrowing species in digesters but abundant in AS (i.e., species belonging to genus Tetrasphaera, $\mathrm{Ca}$. Microthrix, or Dechloromonas) were found in higher relative abundance in MAD treating only AS compared to MAD treating AS and PS. These results underline that besides substrate characteristics, the immigrating bacterial load has a strong impact on the total bacterial microbiome in AD. Additionally, we also observed the influence of feed sludge on archaeal community (Figure S15A), with significant difference between the digesters with different feed sludge (ANOSIM: $\mathrm{R}=0.23, P=$ 0.001 ). It is interesting to see that the species belonging to genus Methanolinea were rare in the digesters only fed with AS (Figure S15B).

\section{Relationship between MAD microbiome and its driving factors predicted by PLS regression model}

We applied PLS regression to predict key operational variables and performance parameters and their relationship to the microbiome in MAD. Separate PLS models were built on the bacterial microbiome at species level and archaeal ASVs with each factor (for bacteria: temperature, OLR, TAN, and biogas yield; for archaea: temperature, TAN, acetate, and biogas yield). Very good prediction accuracy was observed on the bacterial microbiome, where the CV R ${ }^{2}$ of all four PLS models exceeded 0.85 (Fig. 4). However, none of the models based on archaeal ASVs had the $\mathrm{R}^{2}$ over 0.80 , except for biogas yield (Fig. 4). PLS regression models were also carried out on $\mathrm{pH}$ and SRT, since they are important AD parameters (Figure S16 and S17). The growing bacterial species and archaeal ASVs, which most significantly contributed to each PLS regression model (the contribution was estimated using inferential statistics for corresponding regression coefficients, $P<0.05$ ), for both positive and negative contributions, are shown in Fig. 5.

Most growing bacterial species exhibiting significant correlations were represented by novel taxa (Fig. 5A). Species belonging to the same genus were correlated to different operational or performance parameters, such as was the case with two species in family Anaerolineaceae (midas_s_467 and midas_s_1462 belonging to genus midas_g_467). The positive correlation of midas_s_467 with TAN and biogas yield, and the negative 
correlation of midas_s_1462 with temperature and OLR, could explain the abundance variability and trend across MAD (Figure S9). Similar observations were found for three species belonging to genus midas_g_12 (family Prolixibacteraceae, phylum Bacteroidetes). The ecological function of these novel species is unknown so the PLS correlation results may provide hypotheses which could aid the design of experiments to reveal the role of novel taxa in AD [71]. Among the known species, $\mathrm{Ca}$. Brevefilum fementans showed a positive correlation with TAN, which can indicate a preference or tolerance to slightly higher TAN conditions. This hypothesis is supported by their genome blueprint indicating that $\mathrm{Ca}$. B. fementans can ferment proteinaceous substrates to VFAs with ammonium as a by-product of protein degradation [72]. Additionally, species belonging to the known syntrophic genera $\mathrm{Ca}$. Cloacimonas, Smithella, Syntrophomonas, and Syntrophorhabdus were found mostly negatively correlated with TAN and temperature, thereby confirming the high sensitivity of this group to environmental conditions [73-76].

Non-growing bacterial species (primarily immigrating with the feed sludge) showed negative correlations to some key parameters (Figure S18), especially for biogas yield and SRT, suggesting that they are not directly involved in the conversion of feed stocks to biogas and are probably degraded or washed out of the digesters. This is exemplified by Tetrasphaera midas_s_5, the most abundant non-growing species in MAD, and it was negatively correlated with SRT together with Tetrasphaera elongata and midas_s_299. Tetrasphaera is very abundant in Danish wastewater treatment plants [55] and is introduced into the digester with the waste activated sludge. The negative correlation with the SRT indicates that Tetrasphaera is dying off in the digesters despite the potential for surviving or growing under anaerobic conditions as fermenters, and polyphosphate accumulators [77].

Correlation results for archaeal ASVs are shown in Fig. 5B. Generally, ASVs belonging to the same genus show the same trends. For example, ASVs classified to family Methanosarcinaceae and order Methanosarcinales known as acetoclastic and hydogenotrophic methanogens, positively correlated with acetate. This is consistent with other findings [78] where Methanosarcina was most abundant in digesters with higher acetate concentration. In contrast, most Methanothrix ASVs correlated negatively with acetate, supporting the dominance of Methanothrix at low acetate concentrations [79]. Many Methanothrix ASVs also showed negative correlation with TAN, which is in agreement with studies that show that Methanothrix is the methanogen most sensitive to ammonia inhibition [80]. It is interesting that even small TAN variations as seen here for Danish digesters (603-972 mg N/L, MAD - Table 1) can affect individual Methanothrix ASVs in different ways.

For hydrogenotrophic methanogens, different ASVs from the same genus or species showed diverse correlations with AD parameters. The second most abundant archaeal species in MAD belonging to genus Methanolinea (midas_s_1190) included three ASVs, which correlated with TAN differently suggesting that species microdiversity can influence process performance. The negative correlation of many hydrogenotrophic methanogens with biogas yield, such as Methanoculleus, could be due to their ability to 
survive/compete at sub-optimal AD conditions (e.g., increased TAN) [80] which could translate into lower biogas yields.

Overall, the PLS regression models presented enable elucidation of the relationships between important $\mathrm{AD}$ parameters and the main drivers shaping $\mathrm{AD}$ microbiome at very high resolution. The results for known taxa agree with present knowledge, thus verifying the robustness of the PLS application in microbiome study. Importantly, a combination of the PLS regression with species-level microbial data provides the first insight into potential functional importance of several novel microorganisms, where little or no description of their ecology and physiology is available. Based on our observations, hydrolytic-fermentative bacteria, and acetogenic syntrophs along with archaeal methanogens, all have significant and quantitative relationships with important parameters at MAD. This shows great promise for the improved models, and optimized functional performance of AD.

\section{Conclusion}

A six-year survey of 46 anaerobic digesters located at 22 Danish WWTPs provided a comprehensive overview of typical operational and performance parameters, detailed identification of the $\mathrm{AD}$ microbiome at species level, and elucidated relationships between specific taxa and key parameters in AD. The anaerobic digesters surveyed were running stably but operated at low intensity, a common feature across digesters in WWTP. Non-growing species migrating from the feed sludge were abundant in mesophilic and thermophilic AD, but did not seem to contribute to the functionality of AD. In contrast, many growing species were novel and identified using MiDAS 3 taxonomy, and their physiological and ecological roles in $\mathrm{AD}$ remains to be described. The microbiome of the three types of $\mathrm{AD}$ surveyed (mesophilic, thermophilic, and thermal hydrolysis pretreatment-mesophilic) showed high stability within plants, forming separate clusters for all bacteria, growing bacteria, and archaea depending on the operational parameters. The variations of growing bacteria within mesophilic digesters were related to organic loading rate, ammonium concentration, feed sludge characteristics, and biogas yield. Multiple correlations between growing bacteria and archaea at species level and key parameters were found, forming a basis for future studies of the ecology and function of novel taxa. 


\section{Figures}

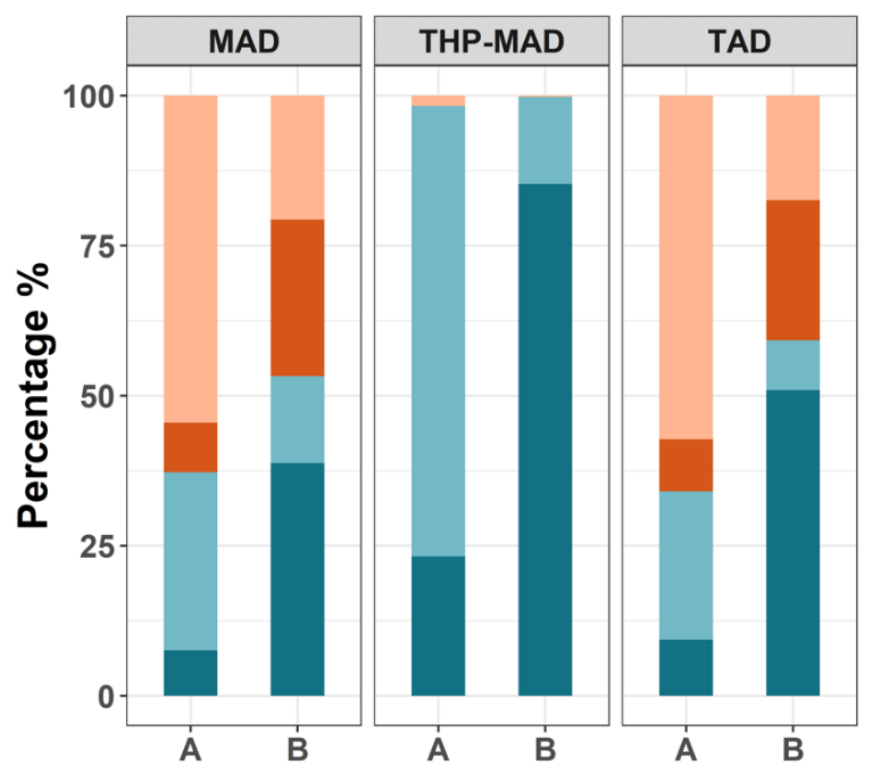

ASV

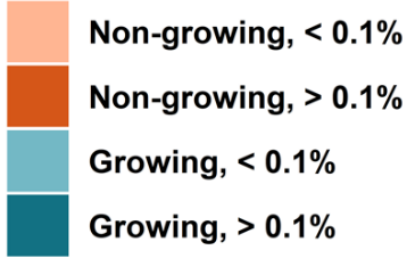

A: ASV counts

B: ASV relative abundance

528 Fig. 1 Composition of growing and non-growing ASVs in Danish ADs at WWTPs.

529 The total ASVs (median relative abundance $>0.01 \%$ ) divided into four groups based on 530 growth ratio (growing/non-growing) and relative abundance (high/low abundant, $0.1 \%$ 531 indicates the cutoff value). A and B show the composition of ASV counts and ASV 532 relative abundance for these four groups in MAD, THP-MAD, and TAD, respectively. $533 \mathrm{MAD}=$ Mesophilic AD, THP = Mesophilic with thermal hydrolysis pretreatment, TAD $534=$ Thermophilic AD. 


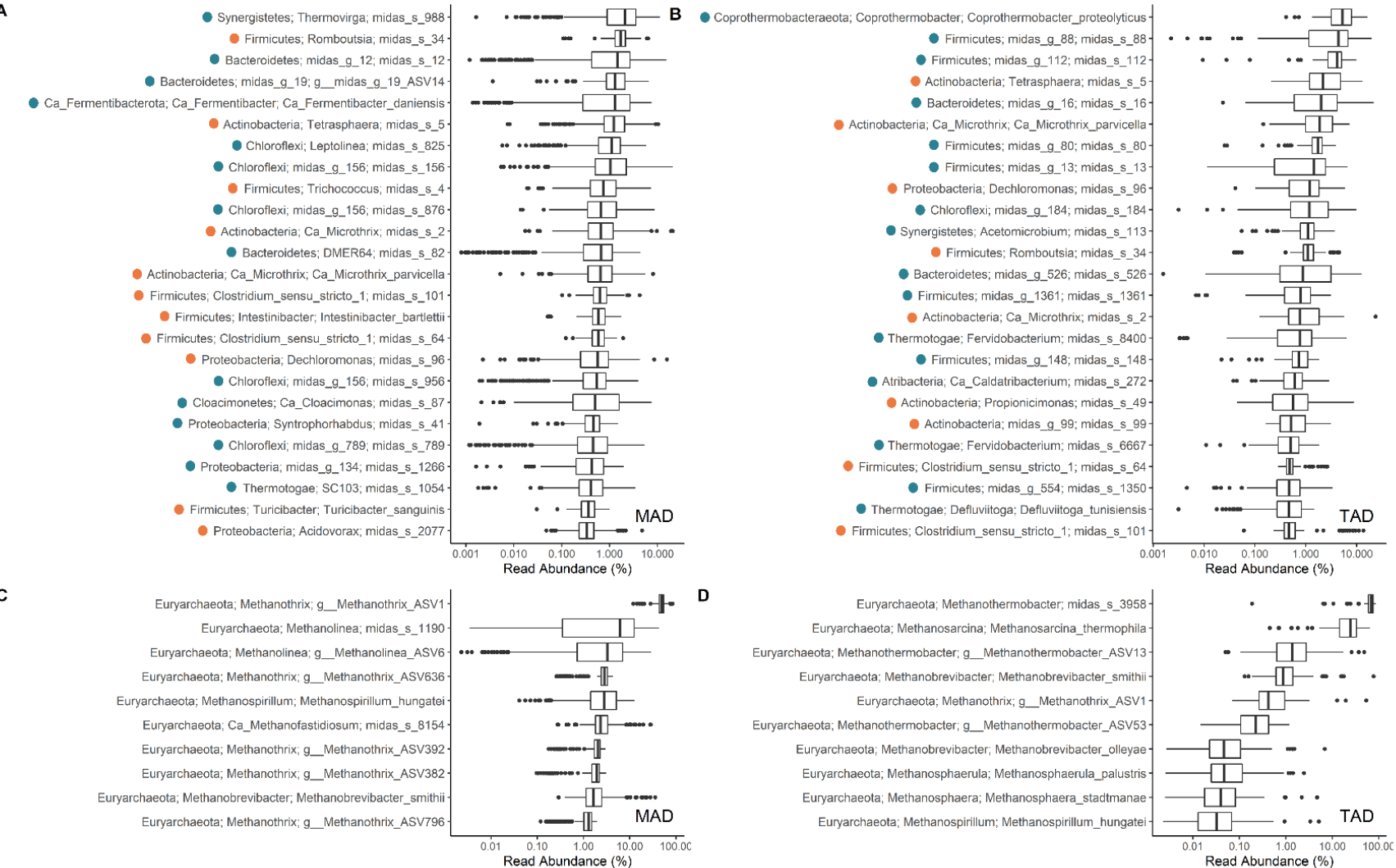

Fig. 2 Boxplots of the most abundant species/ASVs in Danish ADs at WWTPs. (A) The 25 most abundant bacterial species/ASVs in MAD, (B) The 25 most abundant bacteria species/ASVs in TAD, (C) The 10 most abundant archaeal species/ASVs in MAD, (D) The 10 most abundant archaeal species/ASVs in TAD. The dots at the left in A and B indicate whether the species/ASVs are growing (ratio $>10$, blue), non-growing or dying off (ratio $<10$, orange). $\mathrm{MAD}=$ Mesophilic $\mathrm{AD}, \mathrm{TAD}=$ Thermophilic $\mathrm{AD}$. Ratio refers to the digester to influent relative read abundance ratio (please see Additional file 3). Sequences not possessing species-levels classification are shown here as individual ASVs. 

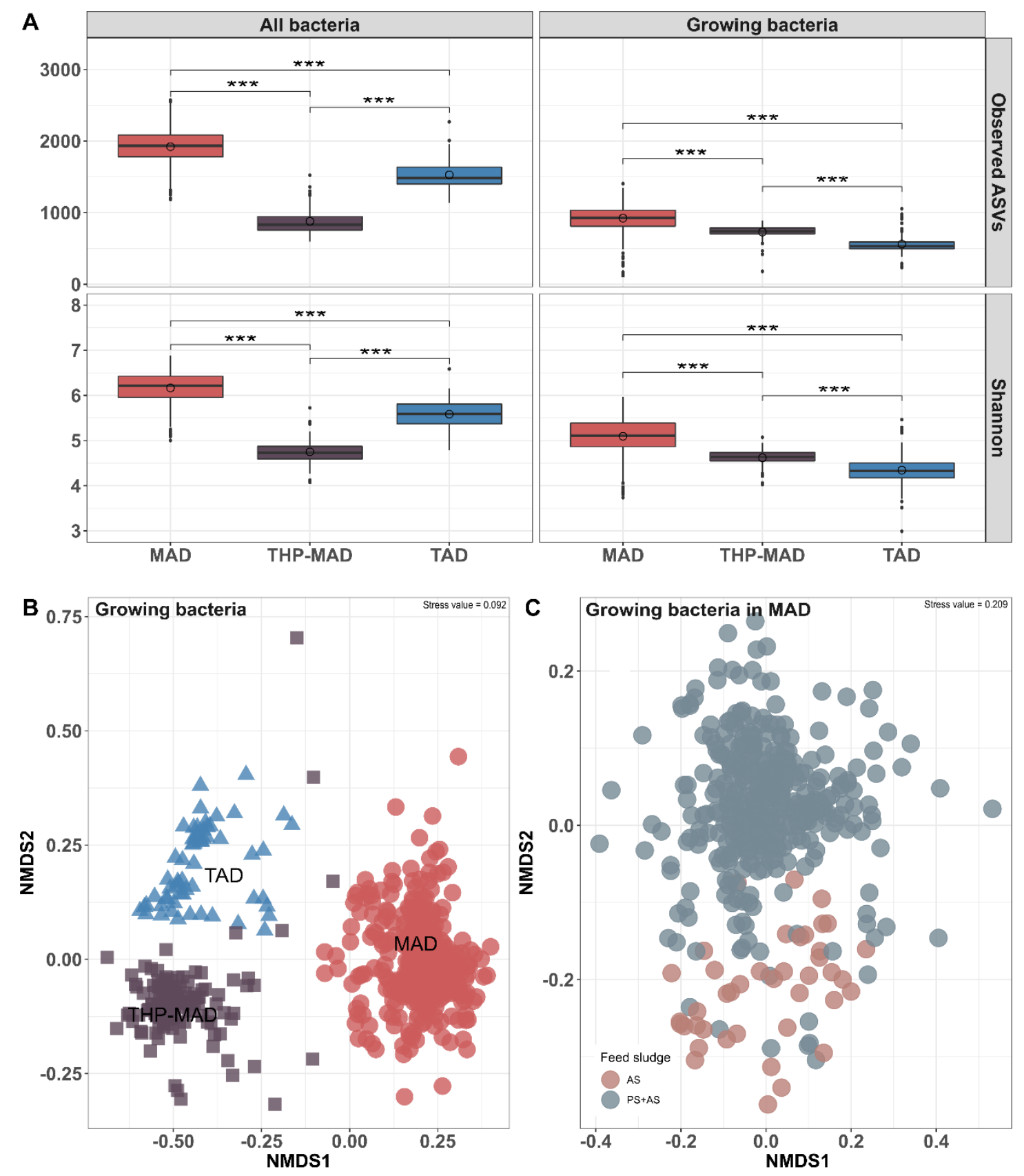

543 Fig. 3 Alpha and beta diversity plots of bacterial and archaeal communities of three

544 types of AD. (A) Boxplots of observed ASVs and Shannon index of entire and growing 545 bacterial community, significant differences are indicated (Wilcoxon rank-sum test; ***, $546 \mathrm{p}<0.001$ ). (B) Non-metric multidimensional scaling (NMDS) plots of growing bacterial 547 community structure based on weighted UniFrac matrix, (C) NMDS plots of growing 548 bacterial community structure of MAD based on weighted UniFrac matrix. MAD = 549 Mesophilic AD, THP-MAD = Mesophilic with thermal hydrolysis pretreatment process, $550 \mathrm{TAD}=$ Mesophilic AD, AS $=$ Activated sludge, $\mathrm{PS}=$ Primary sludge. 

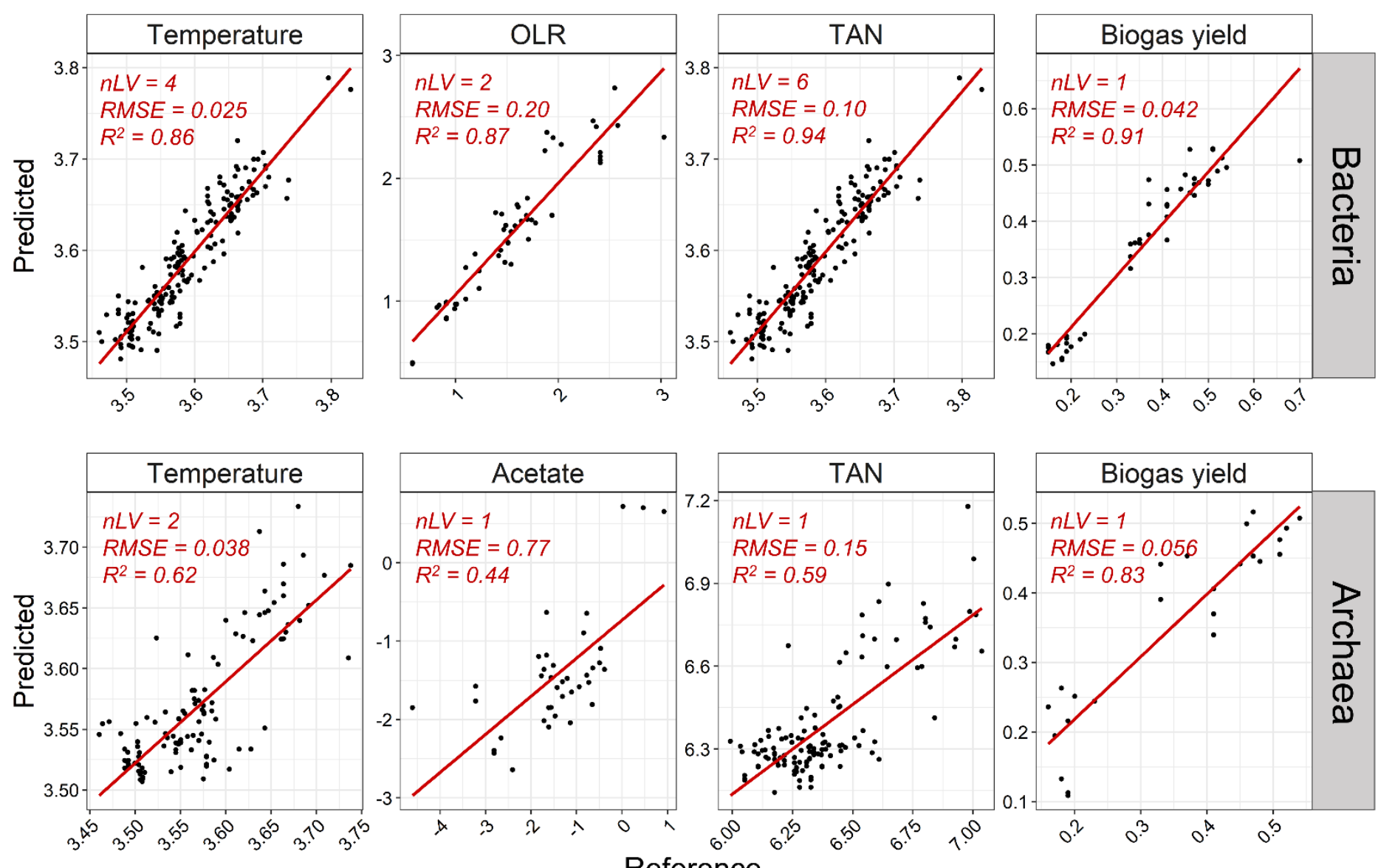

Reference

Fig. 4 Prediction plots of main drivers based on bacterial and archaeal microbiome in MAD by partial least squares regression. MAD $=$ mesophilic $\mathrm{AD}, \mathrm{OLR}=$ organic loading rate, $\mathrm{TAN}=$ total ammonium nitrogen. $\mathrm{nLV}=$ number of selected components, $\mathrm{RMSE}=$ root $\mathrm{mean}$

554 squared error. 
bioRxiv preprint doi: https://doi org/10.1101/2020 06.07.138891; this version posted June 7, 2020. The copyright holder for this preprint (which was not certified by peer review) is the author/funder, who has granted bioRxiv a license to display the preprint in perpetuity. It is made available under aCC-BY-NC-ND 4.0 International license.

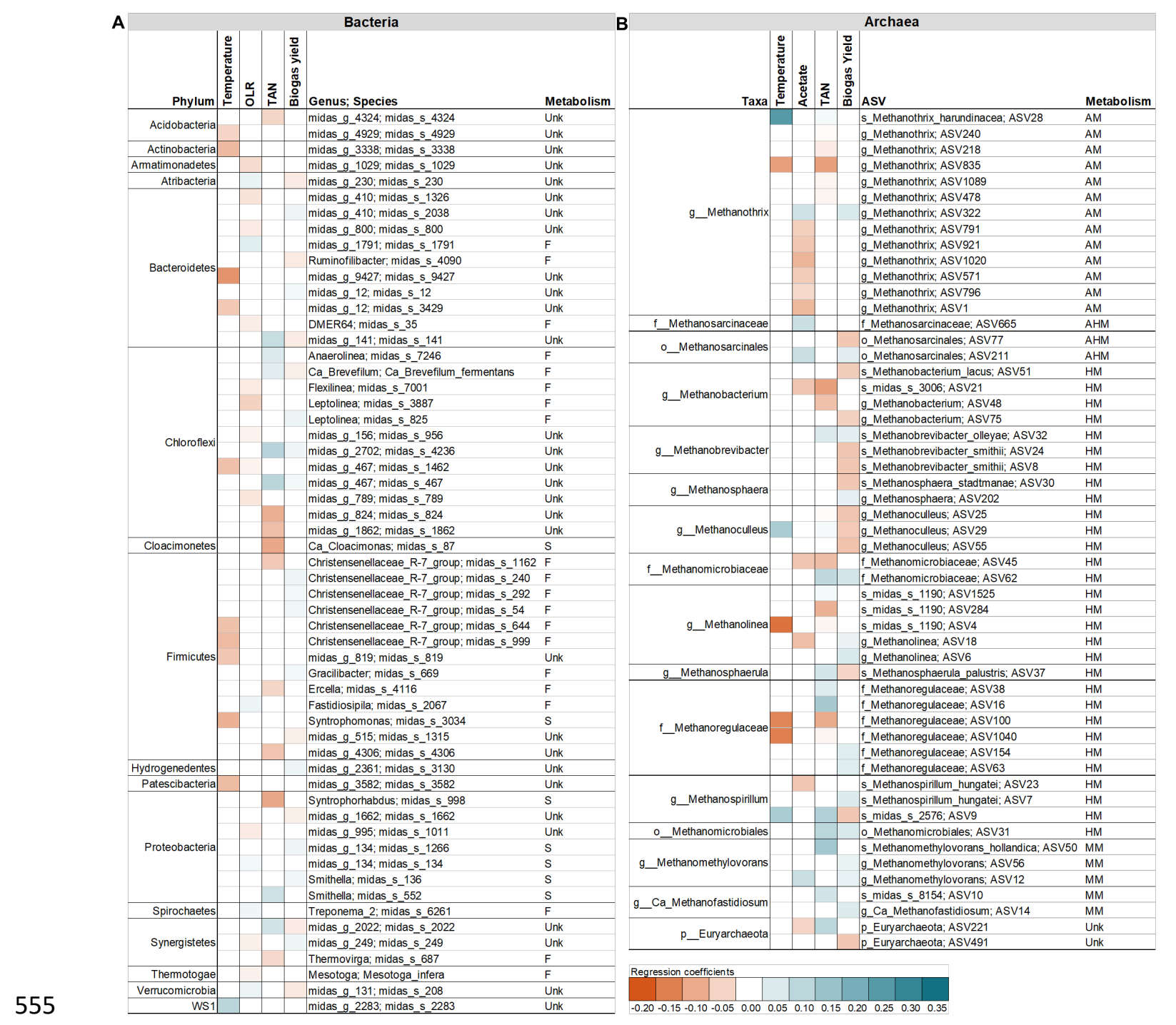

556 Fig. 5 Partial least squares estimation for main driver for important growing 557 bacterial species (A) and archaeal ASVs (B) in MAD. $P<0.05$, positive correlation in 558 blue, negative correlation in orange. $\mathrm{F}=$ Fermenters, $\mathrm{S}=$ Syntrophic bacteria, $\mathrm{AM}=$ 559 Acetoclastic methanogens, AHM = Acetoclastic or Hydrogenotrophic methanogens, HM $560=$ Hydrogenotrophic methanogens, $\mathrm{MM}=$ Methylotrophic methanogens, Unk = Unknown. 


\section{Supplementary information}

562 Additional file 1: Table S1. Overview of WWTP digester capacities, type, and industrial load.

563 Table S2. Linear regression of key variables individually against alpha diversity using the

564 Shannon diversity index at MAD. Table S3. Permutational multivariate analysis (using 565 continuous variables only) of variance of beta diversity using weighted UniFrac matrix at MAD.

566 Figure S1. Comparison of classification between full-length exact sequence variants (FL-ESVs) 567 database and the SILVA_132_SSURef_Nr99 database on the top 50 ASVs in the digester sludge 568 samples from Danish wastewater treatment plants. Figure S2. Box plots of operational and 569 performance parameters of three types of AD. Figure S3. Spearman correlations on operational 570 and performance parameters in AD. Figure S4. Distribution of digester:feed relative read 571 abundance ratios for each ASV. Figure S5. (A) Relative abundance of the 20 most abundant 572 bacterial phyla in AD. (B) Relative abundance of the 50 most abundant bacterial genera in AD (n 573 = 564). Figure S6. Boxplots of the top 100 species/ASVs in MAD. Figure S7. Boxplots of the 574 top 100 species ASVs in TAD. Figure S8. Boxplots of the most abundant species/ASVs in THP575 MAD. Figure S9. Heatmap of the most abundant species/ASVs belonging to the genus T78 in 576 MiDAS 2 (split into the genera midas_g_156 and midas_g_467, all family Anaerolineaceae), 577 genus Ca.Cloacimonas, genus Pelotomaculum, midas_g_995, and genus Methanothrix in Danish 578 digesters at WWTPs. Figure S10. Relative abundance of the 25 most abundant archaeal genera 579 in AD $(\mathrm{n}=402)$. Figure S11. Boxplots of alpha diversity measures of archaeal community of 580 three types of AD. Figure S12. Non-metric multidimensional scaling (NMDS) plots of bacterial 581 and archaeal community structure based on weighted Unifrac matrix colored by WWTPs. Figure 582 S13. Non-metric multidimensional scaling (NMDS) plots of all aacteria and archaea community 583 structures based on weighted Unifrac matrix. Figure S14. (A) Non-metric multidimensional 584 scaling (NMDS) plots of entire bacterial community structure based on weighted UniFrac matrix 585 in MAD. (B) Heatmap of 25 most abundant bacterial species in MAD digesters depending on the 586 composition of feed sludge (AS and PS). Figure S15. (A) Non-metric multidimensional scaling 587 (NMDS) plots of archaeal community structure based on weighted UniFrac matrix in MAD. (B) 588 Heatmap of 15 most abundant archaeal species in MAD digesters depending on the composition 589 of feed sludge (AS and PS). Figure S16. Prediction plots of other important parameters based on 590 bacterial and archaeal microbiome in MAD by partial least square regression. Figure S17. 591 Complete list for partial least squares estimation of key variables with growing bacterial species 592 (A) and archaeal ASVs (B) in MAD. Figure S18. Partial least squares estimation of key variables 593 with non-growing bacterial species in MAD.

594 Additional file 2: Characterization of key parameters of anaerobic digestions

Additional file 3: Digester to influent relative read abundance ratios for each ASV 


\section{Acknowledgements}

597 We thank the operators at different WWTPs in Denmark for providing digester sludge 598 samples and plant records. The Chinese Scientific Research Council is acknowledged for 599 providing financial support to C. Jiang.

\section{Authors' contributions}

601 PHN and CJ conceived and designed the work. CJ, MP, and PHN wrote the manuscript. 602 CJ, MP, SK, and EY performed bioinformatic analysis, statistical analysis, and data 603 visualization. KSA and MSD provided bioinformatics support. MHA, MN, RHK, and LH 604 performed data collection, sampling, and lab procedures. JH and AAH contributed to 605 sample and plant record collection. CJ, MP, PHN, SK, MN, and MSD contributed to data 606 interpretation. All co-authors read and approved the final manuscript.

\section{Funding}

608 This research was funded by Innovation Fund Denmark [NomiGas, grant 1305-00018B]

609 and the Villum Foundation [Illumination of microbial dark matter, grant 13351].

\section{Availability of data and materials}

611 The raw amplicon sequences generated and analyzed during the current study were uploaded to the NCBI Sequence Read Archive (SRA) data depository, with the project number PRJNAXXXXX. Additional data can be shared upon request.

\section{Ethics approval and consent to participate}

615 Not applicable

\section{Consent for publication}

617 Not applicable

\section{Competing interests}

619 The authors declare that they have no competing interests 


\section{Reference}

1. Nielsen PH. Microbial biotechnology and circular economy in wastewater treatment. Microb Biotechnol. 2017;10:1102-5.

2. Puyol D, Batstone DJ, Hülsen T, Astals S, Peces M, Krömer JO. Resource Recovery from Wastewater by Biological Technologies: Opportunities, Challenges, and Prospects. Front Microbiol. Frontiers; 2017;7:2016.

3. Briones A, Raskin L. Diversity and dynamics of microbial communities in engineered environments and their implications for process stability. Curr Opin Biotechnol. 2003;14:270-6.

4. Ofiţeru ID, Lunn M, Curtis TP, Wells GF, Criddle CS, Francis CA, et al. Combined niche and neutral effects in a microbial wastewater treatment community. PNAS. 2010;107:15345-50.

5. Vanwonterghem I, Jensen PD, Dennis PG, Hugenholtz P, Rabaey K, Tyson GW. Deterministic processes guide long-term synchronised population dynamics in replicate anaerobic digesters. ISME J. 2014;8:2015-28.

6. Carballa M, Smits M, Etchebehere C, Boon N, Verstraete W. Correlations between molecular and operational parameters in continuous lab-scale anaerobic reactors. Appl Microbiol Biotechnol. 2011;89:303-14.

7. De Vrieze J, Saunders AM, He Y, Fang J, Nielsen PH, Verstraete W, et al. Ammonia and temperature determine potential clustering in the anaerobic digestion microbiome. Water Res. 2015;75:312-23.

8. Ho D, Jensen P, Batstone D. Effects of temperature and hydraulic retention time on acetotrophic pathways and performance in high-rate sludge digestion. Environ Sci Technol. 2014;48:6468-76.

9. Kim M, Ahn Y-H, Speece RE. Comparative process stability and efficiency of anaerobic digestion; mesophilic vs. thermophilic. Water Res. 2002;36:4369-85.

10. Kirkegaard RH, McIlroy SJ, Kristensen JM, Nierychlo M, Karst SM, Dueholm MS, et al. The impact of immigration on microbial community composition in full-scale anaerobic digesters. Sci Rep. 2017;7:9343.

11. Lucas R, Kuchenbuch A, Fetzer I, Harms H, Kleinsteuber S. Long-term monitoring reveals stable and remarkably similar microbial communities in parallel full-scale biogas reactors digesting energy crops. FEMS Microbiol Ecol. 2015;91(3):fiv004.

12. Vanwonterghem I, Jensen PD, Rabaey K, Tyson GW. Temperature and solids retention time control microbial population dynamics and volatile fatty acid production in replicated anaerobic digesters. Sci Rep. 2015;5:8496. 
13. Mei R, Nobu MK, Narihiro T, Kuroda K, Muñoz Sierra J, Wu Z, et al. Operationdriven heterogeneity and overlooked feed-associated populations in global anaerobic digester microbiome. Water Res. 2017;124:77-84.

14. Schnürer A, Nordberg Å. Ammonia, a selective agent for methane production by syntrophic acetate oxidation at mesophilic temperature. Water Sci Technol. 2008;57:735-40.

15. Sundberg C, Al-Soud WA, Larsson M, Alm E, Yekta SS, Svensson BH, et al. 454 pyrosequencing analyses of bacterial and archaeal richness in 21 full-scale biogas digesters. FEMS Microbiol Ecol. 2013;85:612-26.

16. Mei R, Kim J, Wilson FP, Bocher BTW, Liu W-T. Coupling growth kinetics modeling with machine learning reveals microbial immigration impacts and identifies key environmental parameters in a biological wastewater treatment process. Microbiome. 2019;7:65.

17. Bocher BTW, Cherukuri K, Maki JS, Johnson M, Zitomer DH. Relating methanogen community structure and anaerobic digester function. Water Res. 2015;70:425-35.

18. Morris R, Schauer-Gimenez A, Bhattad U, Kearney C, Struble CA, Zitomer D, et al. Methyl coenzyme $\mathrm{M}$ reductase (mcrA) gene abundance correlates with activity measurements of methanogenic $\mathrm{H} 2 / \mathrm{CO} 2$-enriched anaerobic biomass. Microb Biotechnol. 2014;7:77-84.

19. Tale VP, Maki JS, Struble CA, Zitomer DH. Methanogen community structureactivity relationship and bioaugmentation of overloaded anaerobic digesters. Water Res. 2011;45:5249-56.

20. Venkiteshwaran K, Bocher B, Maki J, Zitomer D. Relating anaerobic digestion microbial community and process function. Microbiol Insights. 2015;8:37-44.

21. Kucheryavskiy S. mdatools - R package for chemometrics. Chemometr Intell Lab. 2020;198:103937.

22. Callahan BJ, McMurdie PJ, Holmes SP. Exact sequence variants should replace operational taxonomic units in marker-gene data analysis. ISME J. 2017;11:2639-43.

23. García-García N, Tamames J, Linz AM, Pedrós-Alió C, Puente-Sánchez F. Microdiversity ensures the maintenance of functional microbial communities under changing environmental conditions. ISME J. 2019;1-15.

24. Dueholm MS, Andersen KS, Petriglieri F, McIlroy SJ, Nierychlo M, Petersen JF, et al. Comprehensive ecosystem-specific 16S rRNA gene databases with automated taxonomy assignment (AutoTax) provide species-level resolution in microbial ecology. bioRxiv. 2019;672873.

25. Nierychlo M, Andersen KS, Xu Y, Green N, Jiang C, Albertsen M, et al. MiDAS 3: An ecosystem-specific reference database, taxonomy and knowledge platform for 
activated sludge and anaerobic digesters reveals species-level microbiome composition of activated sludge. Water Res. 2020;115955.

26. Quast C, Pruesse E, Yilmaz P, Gerken J, Schweer T, Yarza P, et al. The SILVA ribosomal RNA gene database project: improved data processing and web-based tools. Nucleic Acids Res. 2013;41:D590-6.

27. DeSantis TZ, Hugenholtz P, Larsen N, Rojas M, Brodie EL, Keller K, et al. Greengenes, a Chimera-Checked 16S rRNA Gene Database and Workbench Compatible with ARB. Appl Environ Microbiol. 2006;72:5069-72.

28. Cole JR, Wang Q, Fish JA, Chai B, McGarrell DM, Sun Y, et al. Ribosomal Database Project: data and tools for high throughput rRNA analysis. Nucleic Acids Res. 2014;42:D633-42.

29. Hao L, Michaelsen TY, Singleton CM, Dottorini G, Kirkegaard RH, Albertsen M, et al. Novel syntrophic bacteria in full-scale anaerobic digesters revealed by genomecentric metatranscriptomics. ISME J. 2020;1-13.

30. Kirkegaard RH, Dueholm MS, McIlroy SJ, Nierychlo M, Karst SM, Albertsen M, et al. Genomic insights into members of the candidate phylum Hyd24-12 common in mesophilic anaerobic digesters. ISME J. 2016;10:2352-64.

31. Lane DJ. 16S/23S rRNA sequencing. Nucleic Acid Techniques in Bacterial Systematics. New York: Wiley; 1991. p. 125-175.

32. Muyzer G, Waal EC de, Uitterlinden AG. Profiling of complex microbial populations by denaturing gradient gel electrophoresis analysis of polymerase chain reaction-amplified genes coding for 16S rRNA. Appl Environ Microbiol. 1993;59:695-700.

33. Pinto AJ, Raskin L. PCR Biases Distort Bacterial and Archaeal Community Structure in Pyrosequencing Datasets. PLoS One. 2012; 7:e43093.

34. Albertsen M, Karst SM, Ziegler AS, Kirkegaard RH, Nielsen PH. Back to basics The influence of DNA extraction and primer choice on phylogenetic analysis of activated sludge communities. PLoS One. 2015;10:e0132783.

35. Edgar RC. Search and clustering orders of magnitude faster than BLAST. Bioinformatics. 2010;26:2460-1.

36. Edgar RC. UNOISE2: improved error-correction for Illumina 16S and ITS amplicon sequencing. bioRxiv. 2016;081257.

37. Edgar RC. Accuracy of taxonomy prediction for $16 \mathrm{~S}$ rRNA and fungal ITS sequences. PeerJ. 2018;6:e4652.

38. R Core Team. R: A language and environment for statistical computing. $\mathrm{R}$ Foundation for StatisticalComputing, Vienna, Austria.; 2019. 
39. Wickham H. ggplot2: Elegant Graphics for Data Analysis. Springer-Verlag New York; 2016.

40. Bastian M, Heymann S, Jacomy M. Gephi: An Open Source Software for Exploring and Manipulating Networks. Third International AAAI Conference on Weblogs and Social Media. 2009.

41. Caporaso JG, Kuczynski J, Stombaugh J, Bittinger K, Bushman FD, Costello EK, et al. QIIME allows analysis of high-throughput community sequencing data. Nat Methods. 2010;7:335-6.

42. Martens H, Martens M. Modified Jack-knife estimation of parameter uncertainty in bilinear modelling by partial least squares regression (PLSR). Food Qual Prefer. 2000;11:5-16.

43. Calusinska M, Goux X, Fossépré M, Muller EEL, Wilmes P, Delfosse P. A year of monitoring 20 mesophilic full-scale bioreactors reveals the existence of stable but different core microbiomes in bio-waste and wastewater anaerobic digestion systems. Biotechnol Biofuels. 2018;11:196.

44. Mata-Alvarez J, Dosta J, Romero-Güiza MS, Fonoll X, Peces M, Astals S. A critical review on anaerobic co-digestion achievements between 2010 and 2013. Renew Sustain Energy Rev. 2014;36:412-27.

45. Martí-Herrero J, Soria-Castellón G, Diaz-de-Basurto A, Alvarez R, Chemisana D. Biogas from a full scale digester operated in psychrophilic conditions and fed only with fruit and vegetable waste. Renew Energy. 2019;133:676-84.

46. Fotidis IA, Wang H, Fiedel NR, Luo G, Karakashev DB, Angelidaki I. Bioaugmentation as a solution to increase methane production from an ammonia-rich substrate. Environ Sci Technol. 2014;48:7669-76.

47. Luo G, Fotidis IA, Angelidaki I. Comparative analysis of taxonomic, functional, and metabolic patterns of microbiomes from 14 full-scale biogas reactors by metagenomic sequencing and radioisotopic analysis. Biotechnol Biofuels. 2016;9:51.

48. Chen Y, Cheng JJ, Creamer KS. Inhibition of anaerobic digestion process: A review. Bioresour Technol. 2008;99:4044-64.

49. Tsapekos P, Kougias PG, Treu L, Campanaro S, Angelidaki I. Process performance and comparative metagenomic analysis during co-digestion of manure and lignocellulosic biomass for biogas production. Appl Energy. 2017;185:126-35.

50. Petriglieri F, Nierychlo M, Nielsen PH, McIlroy SJ. In situ visualisation of the abundant Chloroflexi populations in full-scale anaerobic digesters and the fate of immigrating species. Nerenberg R, editor. PLoS One. 2018;13:e0206255.

51. Lee J, Kim E, Han G, Tongco JV, Shin SG, Hwang S. Microbial communities underpinning mesophilic anaerobic digesters treating food wastewater or sewage sludge: A full-scale study. Bioresour Technol. 2018;259:388-97. 
52. Lee S-H, Kang H-J, Haeng Lee Y, Jun Lee T, Han K, Choi Y, et al. Monitoring bacterial community structure and variability in time scale in full-scale anaerobic digesters. J Environ Monit. 2012;14:1893-905.

53. Nelson MC, Morrison M, Yu Z. A meta-analysis of the microbial diversity observed in anaerobic digesters. Bioresour Technol. 2011;102:3730-9.

54. Rivière D, Desvignes V, Pelletier E, Chaussonnerie S, Guermazi S, Weissenbach J, et al. Towards the definition of a core of microorganisms involved in anaerobic digestion of sludge. ISME J. 2009;3:700-14.

55. Stokholm-Bjerregaard M, McIlroy SJ, Nierychlo M, Karst SM, Albertsen M, Nielsen PH. A critical assessment of the microorganisms proposed to be important to enhanced biological phosphorus removal in full-scale wastewater treatment systems. Front Microbiol. 2017;8:718.

56. McIlroy SJ, Kristiansen R, Albertsen M, Karst SM, Rossetti S, Nielsen JL, et al. Metabolic model for the filamentous 'Candidatus Microthrix parvicella' based on genomic and metagenomic analyses. ISME J. 2013;7:1161-72.

57. Pelletier E, Kreimeyer A, Bocs S, Rouy Z, Gyapay G, Chouari R, et al. "Candidatus Cloacamonas Acidaminovorans": Genome Sequence Reconstruction Provides a First Glimpse of a New Bacterial Division. J Bacteriol. 2008;190:2572-9.

58. McIlroy SJ, Kirkegaard RH, McIlroy B, Nierychlo M, Kristensen JM, Karst SM, et al. MiDAS 2.0: an ecosystem-specific taxonomy and online database for the organisms of wastewater treatment systems expanded for anaerobic digester groups. Database. 2017;2017:bax016.

59. Leclerc M, Delgènes J-P, Godon J-J. Diversity of the archaeal community in 44 anaerobic digesters as determined by single strand conformation polymorphism analysis and 16S rDNA sequencing. Environ Microbiol. 2004;6:809-19.

60. Hori T, Haruta S, Ueno Y, Ishii M, Igarashi Y. Dynamic Transition of a Methanogenic Population in Response to the Concentration of Volatile Fatty Acids in a Thermophilic Anaerobic Digester. Appl Environ Microbiol. 2006;72:1623-30.

61. Choi J-M, Han S-K, Lee C-Y. Enhancement of methane production in anaerobic digestion of sewage sludge by thermal hydrolysis pretreatment. Bioresour Technol. 2018;259:207-13.

62. Wandera SM, Westerholm M, Qiao W, Yin D, Jiang M, Dong R. The correlation of methanogenic communities' dynamics and process performance of anaerobic digestion of thermal hydrolyzed sludge at short hydraulic retention times. Bioresour Technol. 2019;272:180-7.

63. Karakashev D, Batstone DJ, Angelidaki I. Influence of environmental conditions on methanogenic compositions in anaerobic biogas reactors. Appl Environ Microbiol. $2005 ; 71: 331-8$. 
64. Levén L, Eriksson ARB, Schnürer A. Effect of process temperature on bacterial and archaeal communities in two methanogenic bioreactors treating organic household waste. FEMS Microbiol Ecol. 2007;59:683-93.

65. Li Y-F, Nelson MC, Chen P-H, Graf J, Li Y, Yu Z. Comparison of the microbial communities in solid-state anaerobic digestion (SS-AD) reactors operated at mesophilic and thermophilic temperatures. Appl Microbiol Biotechnol. 2015;99:969-80.

66. Goux X, Calusinska M, Lemaigre S, Marynowska M, Klocke M, Udelhoven T, et al. Microbial community dynamics in replicate anaerobic digesters exposed sequentially to increasing organic loading rate, acidosis, and process recovery. Biotechnol Biofuels. 2015;8:122.

67. Xu R, Yang Z-H, Zheng Y, Liu J-B, Xiong W-P, Zhang Y-R, et al. Organic loading rate and hydraulic retention time shape distinct ecological networks of anaerobic digestion related microbiome. Bioresour Technol. 2018;262:184-93.

68. Razaviarani V, Buchanan ID. Reactor performance and microbial community dynamics during anaerobic co-digestion of municipal wastewater sludge with restaurant grease waste at steady state and overloading stages. Bioresour Technol. 2014;172:232-40.

69. Gou C, Yang Z, Huang J, Wang H, Xu H, Wang L. Effects of temperature and organic loading rate on the performance and microbial community of anaerobic codigestion of waste activated sludge and food waste. Chemosphere. 2014;105:146-51.

70. Vanwonterghem I, Jensen PD, Ho DP, Batstone DJ, Tyson GW. Linking microbial community structure, interactions and function in anaerobic digesters using new molecular techniques. Curr Opin Biotechnol. 2014;27:55-64.

71. Peces M, Astals S, Jensen PD, Clarke WP. Deterministic mechanisms define the long-term anaerobic digestion microbiome and its functionality regardless of the initial microbial community. Water Res. 2018;141:366-76.

72. McIlroy SJ, Kirkegaard RH, Dueholm MS, Fernando E, Karst SM, Albertsen M, et al. Culture-Independent Analyses Reveal Novel Anaerolineaceae as Abundant Primary Fermenters in Anaerobic Digesters Treating Waste Activated Sludge. Front Microbiol. 2017;8:1134.

73. Hao L, Bize A, Conteau D, Chapleur O, Courtois S, Kroff P, et al. New insights into the key microbial phylotypes of anaerobic sludge digesters under different operational conditions. Water Res. 2016;102:158-69.

74. Ariesyady H, Ito T, Okabe S. Functional bacterial and archaeal community structures of major trophic groups in a full-scale anaerobic sludge digester. Water Res. 2007;41:1554-68. 
75. Ariesyady HD, Ito T, Yoshiguchi K, Okabe S. Phylogenetic and functional diversity of propionate-oxidizing bacteria in an anaerobic digester sludge. Appl Microbiol Biotechnol. 2007;75:673-83.

76. Narihiro T, Nobu MK, Kim N-K, Kamagata Y, Liu W-T. The nexus of syntrophyassociated microbiota in anaerobic digestion revealed by long-term enrichment and community survey. Environ Microbiol. 2015;17:1707-20.

77. Kristiansen R, Nguyen HTT, Saunders AM, Nielsen JL, Wimmer R, Le VQ, et al. A metabolic model for members of the genus Tetrasphaera involved in enhanced biological phosphorus removal. ISME J. 2013;7:543-54.

78. Jetten MSM, Stams AJM, Zehnder AJB. Methanogenesis from acetate: a comparison of the acetate metabolism in Methanothrix soehngenii and Methanosarcina spp. FEMS Microbiol Rev. 1992;8:181-97.

79. Gehring T, Niedermayr A, Berzio S, Immenhauser A, Wichern M, Lübken M. Determination of the fractions of syntrophically oxidized acetate in a mesophilic methanogenic reactor through an $12 \mathrm{C}$ and $13 \mathrm{C}$ isotope-based kinetic model. Water Res. 2016;102:362-73.

80. Capson-Tojo G, Moscoviz R, Astals S, Robles Á, Steyer J-P. Unraveling the literature chaos around free ammonia inhibition in anaerobic digestion. Renew Sustain Energy Rev. 2020;117:109487. 